\title{
ORIGINAL
}

\section{DEPENDENCIA Y NECESIDADES DE CUIDADOS NO CUBIERTAS DE LAS PERSONAS MAYORES DE UNA ZONA DE SALUD DE ZARAGOZA (*)}

\author{
Concepción Tomás Aznar (1), LA Moreno Aznar (1), C. Germán Bes (1), T. Alcalá Nalváiz (2) y E. \\ Andrés Esteban (2). \\ (1) Escuela Universitaria de Ciencias de la Salud. Universidad de Zaragoza. \\ (2) Departamento de Estadística e Investigación Operativa. Universidad de Zaragoza. \\ (*) Este trabajo se realizó con un proyecto de investigación financiado por CONSI+D (PCM 4394)
}

\section{RESUMEN}

Fundamento: El nivel de autonomía para las actividades del cuidado personal en personas mayores y la necesidad de apoyo no cubiertas dependen de diferentes factores poco conocidos en nuestro medio. Los cuidados en la dependencia se facilitan principalmente por el sistema informal de cuidados. El propósito del estudio es estimar la prevalencia de dependencia para las actividades del cuidado personal y los factores asociados, e identificar los factores asociados con las necesidades de cuidados no cubiertas en personas dependientes, en población mayor de 75 años de Zaragoza.

Método: Se realizó un estudio transversal en una muestra representativa de la población no institucionalizada mayor de 75 años, en una Zona de Salud de Zaragoza, en el año 1998 $(\mathrm{n}=351)$. Los datos se cumplimentaron a través de una entrevista directa. Se utilizó el índice de Katz para valorar la capacidad funcional para las actividades del cuidado personal y un cuestionario ad hoc para el apoyo informal y el resto de variables. El apoyo informal, las características sociodemográficas, autopercepción de salud y depresión (Escala de Depresión de Yesavage) se consideraron como variables independientes. Se aplicó la regresión logística para identificar los factores asociados a la dependencia y necesidades de cuidados no cubiertas.

Resultados: La prevalencia de dependencia para las actividades del cuidado personal es de un 37,3\%. Las variables que predicen la dependencia para las actividades del cuidado personal son una mala percepción de salud, depresión establecida, ser mujer y ser mayor de 85 años. La prevalencia de necesidades de apoyo no cubiertas en personas dependientes para las actividades del cuidado personal es de $22,1 \%$. El apoyo informal lo presta la familia. Ser mujer y vivir sola se presenta como un factor de riesgo para no tener las necesidades cubiertas, así como también no estar deprimido.

Conclusiones: El porcentaje de personas mayores con dependencia es elevado. El apoyo informal es insuficiente y limitado a la familia. El modelo de atención a las personas mayores en España debería desarrollar servicios de apoyo emocional, económicos y sociales a la familia y establecer una especial atención sobre las personas con dependencia que viven solas y con escasos recursos materiales.

Palabras clave: dependencia, actividades de la vida diaria, cuidados informales, necesidades no cubiertas, Ancianos.

Correspondencia:

Concepción Tomás Aznar

Escuela Universitaria de Ciencias de la Salud

Universidad de Zaragoza

C/ Domingo Miral, s/n 50009 Zaragoza

Correo electrónico: ctomas@posta.unizar.es

\section{ABSTRACT}

\section{Dependency and Unmet Need of Care in Older People in a Health Area of Saragossa, Spain}

Background: Dependency to carry out the activities of daily living (ADL) and home care needs of older adults depend upon factors which are not well understood. Caregiving is mainly provided by the family. The aim of this study is to estimate the prevalence of ADL dependence and associated factors among older adults and to identify associated factors with unmet home care needs in people over age 75 in Zaragoza (Spain).

Method: A cross-sectional study was conducted on a representative sample of the community dwelling population over age 75 in one Health Care District in Saragossa in 1998 $(\mathrm{n}=351)$. Data were collected during a personal interview. ADL dependency was assessed by the Katz index.Home care needs were assesed using prevalent norms for desired frequency of care. Sources of informal care, socio-demographic characteristics, self-rated health and depression (Yesavage Geriatric Depression Scale) were considered independentvariables. Logistic regression was used to identify factors associated with both ADL dependency and unmet home care needs.

Results: Prevalence of ADL dependence is $37.3 \%$. The variables predicting ADL dependence are the perception of poor health, depression, being a female and being over 85 years of age. The prevalence of unmet care needs among ADL dependent individuals is $22.1 \%$. Sources of help are limited to the families. Being a woman and living alone are the stronger risk factors for unmet needs.

Conclusions: A high percentage of older adults are dependent. Informal support is insufficient and confined to the family. The social and health care system in Spain should provide to the family, emotional, financial and social support and special care for disabled people living alone and with limited material resources.

Keywords: Dependency. Activities of daily living.Informal care. Unmet need. Elderly. 


\section{INTRODUCCIÓN}

El envejecimiento y el mantenimiento de la calidad de vida se perciben, la primera como una realidad evidente en nuestra sociedad y la segunda como un deseo manifestado tanto por las personas como por el propio sistema de salud. El aumento de la esperanza de vida es un objetivo alcanzado por los países más desarrollados. En el último siglo la esperanza de vida ha aumentado 30 años, aunque la velocidad ha sido mayor en las últimas décadas del siglo $\mathrm{Xx}^{1}$. Una realidad asociada al envejecimiento es que el número de personas con dependencia aumenta con la edad ${ }^{2}$ derivándose de ello un aumento en la demanda de cuidados por los procesos crónicos y degenerativos que lleva asociados, fundamentalmente del sistema informal. Los retos que se plantean desde el ámbito sanitario entre otros son: mantener la calidad de vida y su autonomía, promover un envejecimiento activo ${ }^{1}$, desarrollar el sistema formal e informal de cuidados, garantizar la cobertura de las necesidades y aportar apoyo emocional a las personas mayores y a sus cuidadores.

La capacidad funcional es un concepto multidimensional que incluye componentes físicos, psicológicos y sociales. En las personas mayores una buena capacidad funcional indica un alto nivel de independencia, mejor calidad de vida y bienestar en relación a las enfermedades ${ }^{3,4}$ siendo un predictor importante de la necesidad de apoyo ${ }^{5}$.

El apoyo social o apoyo informal se puede considerar como la presencia real o percibida de apoyo, dispensada por parte de la familia, amigos y red social para satisfacer unas necesidades básicas a la persona ${ }^{6,7} . \mathrm{El}$ apoyo informal juega un importante papel en los cuidados en personas mayores dependientes $^{8}$. La influencia de la red de apoyo se ha puesto de manifiesto a diferentes niveles. En el Informe de Canadá se asocia a un mejor estado de salud ${ }^{9}$, otros estudios lo centran en los efectos sobre la mortalidad, las personas que disponen de un buen nivel de apoyo tienen menor riesgo de muerte ${ }^{10,11}$. En otros trabajos la falta de apoyo en varones se asocia a un peor estado de salud percibida ${ }^{12}$, en otros la presencia de apoyo social se considera que tiene un efecto protector frente a la demencia ${ }^{13,14}$, o que mejora la función cognitiva ${ }^{15}$.

Diferentes estudios han puesto de manifiesto la existencia de necesidades de cuidados no cubiertas en personas con dependencia relacionadas con el tipo de convivencia, la edad o la situación económica ${ }^{16-20}$. Las consecuencias negativas de estas necesidades no cubiertas se han centrado en un aumento de depresión, de mortalidad o de incapacidad funcional, asi como de un incremento de la institucionalización y de la utilización de recursos médicos y de enfermería $^{16,20}$. De ahí la importancia de evaluarlas y desarrollar medidas para reducirlas ${ }^{21,22}$. La valoración de las necesidades de cuidados no cubiertas es esencial para la planificación de los servicios de larga duración. Este aspecto no ha sido muy estudiado en la mayoría de los países ni existe hasta el momento información en España que ponga en evidencia estas necesidades no cubiertas.

En España, el nivel de autonomía para las actividades de la vida diaria (AVD) en personas mayores de 65 años es, según la Encuesta Nacional de Salud del año 1997, de más del $75 \%$ respecto a las capacidades de autocuidado, aunque respecto a la movilidad un $11,9 \%$ de las personas encuestadas manifiesta dificultades para andar una hora seguida.

La atención y cuidado a las personas mayores en España tiene características diferenciales respecto a otros países. El modelo social de cuidados y atención a las personas mayores tiene un claro componente familiar, como se describe en el informe del IMSERSO y se considera como un deber moral el cuidado de los mayores ${ }^{23}$. Un $84 \%$ de las personas entrevistadas muestran su acuerdo respecto al cuidado de los mayores y consideran que es una obligación de los 
hijos; un 78\% no están de acuerdo sobre la posibilidad de que sus padres vivan en residencias $^{23}$. La realidad demográfica emergente en nuestra sociedad con un creciente porcentaje de personas mayores, nos obliga a conocer en primer lugar cuál es su grado de dependencia para las actividades del cuidado personal y en segundo lugar cuál es el nivel de apoyo informal disponible por esa población y sus necesidades no cubiertas, para poder planificar adecuadamente el cuidado a los mayores.

El estudio realizado se localiza en una Zona Básica de Salud de Zaragoza, Sta. Lucía, que se caracteriza por presentar una población muy envejecida, con un porcentaje de personas mayores de 75 años de 5,33\% en varones y $10,06 \%$ en mujeres, superando en un $7 \%$ a la de Zaragoza en su conjunto, según el censo de 1991, así como un bajo nivel de instrucción, alto nivel de desempleo y elevado porcentaje de jubilados y pensionistas.

Los objetivos de este trabajo son: 1) estimar la prevalencia de la dependencia en las actividades del cuidado personal en personas mayores de 75 años de la Zona de Salud de Sta. Lucía del Casco Viejo de Zaragoza e identificar los factores asociados con la misma y 2) estimar la proporción de personas con dependencia en las actividades del cuidado personal que no tienen sus necesidades cubiertas y describir sus características.

\section{SUJETOS Y MÉTODOS}

El diseño corresponde a un estudio observacional descriptivo y transversal de la población no institucionalizada mayor de 75 años de la Zona Básica de Salud «Sta. Lucía», correspondiente al casco antiguo de Zaragoza capital. Se llevó a cabo en el año 1998. Para la selección de la muestra se tomó como referencia la tarjeta sanitaria. No se utilizó el último censo debido a que la población de la zona de salud no coincide con los distritos establecidos por el municipio. La población mayor de 75 años con tarjeta sanitaria perteneciente a la zona de salud de nuestro estudio es de 2.988 personas, 2.025 mujeres y 963 hombres. A partir del listado de tarjeta sanitaria se realizó un muestreo aleatorio estratificado por edad y sexo, teniendo en cuenta tres grupos de edad: de $75-79$, de $80-84$ y mayores de 85 años, que incluyó a 455 mujeres y a 274 hombres. De éstos cumplían los criterios de admisión 300 mujeres y 154 hombres; el resto no los cumplía por traslado de domicilio, estar ingresados en alguna institución o por haber fallecido. De las personas seleccionadas aceptaron participar en el estudio 228 mujeres y 123 hombres, lo que supuso una proporción de respuestas de $76 \%$ para las mujeres y de $80 \%$ para los hombres.

La información se obtuvo a través de una entrevista personal en el domicilio mediante una encuesta que se elaboró a partir de cuestionarios utilizados en la Encuesta de Salud de Cataluña ${ }^{12}$, los aplicados por el IMSERSO $^{23}$, y otros de elaboración propia. La captación de las personas se realizó a través del envío de una carta y tres llamadas telefónicas para la localización. Se solicitó su consentimiento para participar en el estudio y se estableció el momento de la cita para la entrevista.

En el cuestionario se incluyeron datos sociodemográficos como: edad, sexo, estado civil, situación de convivencia, nivel de estudios, estableciendo siete categorías, y última ocupación desempeñada, tomando como referencia la clasificación propuesta por la Sociedad Española de Epidemiología para la medida de la clase social a través de la ocupación. Esta clasificación establece los siguientes grupos 1. Directivos y profesiones asociadas a $2 .^{\circ}$ y $3^{\text {er }}$ ciclo universitario; 2. Directivos $<10$ trabajadores y profesiones asociadas a $1^{\text {er }}$ ciclo universitario; 3 . Empleados de tipo administrativo. Trabajadores por cuenta propia; 4. Trabajadores manuales cualificados; 5. Trabajadores semicualificados; 6. Trabajadores no cualificados, añadiendo en nuestro estudio la categoría 7. Amas de $\operatorname{casa}^{24}$. Para el análisis 
multivariante esta variable se agrupó en trabajadores no manuales, manuales y amas de casa y la variable nivel de estudios en primaria incompleta, completa y bachiller $y$ más.

La capacidad funcional se valoró a través del índice de KATZ para evaluar la dependencia para las actividades del cuidado personal $(\mathrm{ACP})^{25}$. La percepción de salud se midió a través de la cuestión, ¿cómo piensa que es su salud?: muy buena, buena, regular, mala y muy mala, agrupándose en tres categorías para el análisis multivariante y el nivel de depresión utilizando el cuestionario de Yesavage.

Sobre la red de apoyo social disponible se preguntó sobre la persona de apoyo principal (PAP) y dos cuestiones sobre apoyo social utilizadas en la Encuesta de Salud de Barcelona, sobre la posibilidad de contar con ayuda en caso de necesitarla ${ }^{12}$.

Las variables dependientes en este trabajo son la «dependencia para realizar las ACP» y las «necesidades de cuidados no cubiertas para las $\mathrm{ACP} »$. Se consideró que una persona era dependiente en las ACP cuando respondía que necesitaba ayuda para realizar algunas de las siguientes actividades: asearse, vestirse, comer, deambulación o ir al WC.

La definición de «necesidades no cubiertas» que propone Williams ${ }^{20}$ se refiere a la atención inadecuada o insuficiente que se recibe por parte de otras personas, en nuestro estudio consideramos «necesidad no cubierta para las ACP» cuando las personas con dependencia para las ACP manifiestan que no reciben ayuda para esas actividades. Para la medida de éstas se elaboró un cuestionario específico que hacía referencia a las distintas ACP (asearse, vestirse, comer, deambulación, ir al WC, ser continente), y se preguntó la frecuencia de recepción de la ayuda según si se recibía: todos los días, tres o cuatro veces por semana, cada dos semanas, cada cuatro semanas o nunca, especificando en cada una de ellas quién realiza el apoyo. Se agruparon para el análisis multivariante en «necesidades no cubiertas» cuando la frecuencia del apoyo era inferior a semanal o no tenían apoyo y como «necesidades cubiertas» cuando el apoyo era diario o de varias veces por semana ya que se refiere a actividades del cuidado personal.

Se describen las variables socioeconómicas y de convivencia, las de percepción de salud, de dependencia para las actividades del cuidado personal y de apoyo social.

Para estudiar la asociación de la variable dependiente «dependencia para las $\mathrm{ACP}$ » con el resto de variables sociodemográficas, se realizó un análisis bivariante y se aplicó el test de chi ${ }^{2}$ con nivel de significación $\mathrm{p}<$ 0,05 y un análisis multivariante ajustando una regresión logística para evaluar las asociaciones de las variables independientes y la dependencia para las ACP.

En el análisis de las «necesidades de cuidados no cubiertas» fue necesario reagrupar las variables sociodemográficas debido al limitado número de observaciones. La edad, se reagrupó en dos categorías y dada la estrecha asociación entre vivir solo y ser mujer, se creó una sola variable con tres categorías: hombres que viven acompañados, mujeres que viven acompañadas y mujeres que viven solas. Se eliminó del análisis el único hombre dependiente que vivía solo. Se realizó un análisis bivariante y se aplicó el test de chi ${ }^{2}$ con nivel de significación $\mathrm{p}<0,05$ y un análisis multivariante ajustando una regresión logística para evaluar las asociaciones de las variables independientes con las necesidades de cuidados no cubiertas.

Se utilizó como base de datos el programa informático Excel y el análisis estadístico de los resultados se realizó con el programa SPSS 9.0.

\section{RESULTADOS}

El número total de personas entrevistadas fue $351,123(35,04 \%)$ varones y 228 
$(64,96 \%)$ mujeres, mayores de 75 años. En un $77,4 \%$ de las entrevistas respondió la persona mayor, un 7,4\% la acompañante y en un $15,2 \%$ ambas.

Respecto al estado civil, lugar de residencia y situación de convivencia, el $41,6 \%$ de las personas estaban casadas, y el $44,7 \%$ viudos, de ellos 129 mujeres. Un $75,2 \%$ vivían habitualmente en su domicilio. Un $20,9 \%$ vivían solas, $38,7 \%$ con su esposa o marido, $28,1 \%$ con los hijos y $9,2 \%$ con otros familiares.

En relación al nivel de estudios, 5,7\% no sabían leer ni escribir, 10,6\% sin estudios, $24,3 \%$ primaria incompleta, $46,6 \%$ primaria completa, Bachiller superior o FP $8 \%$, diplomatura universitaria 3,7\% y licenciatura $1,1 \%$. Respecto a la ocupación la mitad de las mujeres (99/228) habían sido amas de casa, y la casi totalidad restante, trabajadoras no cualificadas y semi-cualificadas; en varones, el primer grupo estaba representado por trabajadores cualificados, seguido por el de semi-cualificados y no cualificados.

Sobre su percepción de salud, 7,9\% pensaban que era muy buena, 51,5\% buena, $29,9 \%$ regular, $8,5 \%$ mala, y $2,1 \%$, muy mala. Un $31,1 \%$ presentaban depresión leve y un 14,5 depresión establecida según el cuestionario de Yesavage.

La prevalencia de dependencia para las ACP fue de un 37,3\%. Del total de mujeres, un $44,7 \%$ eran dependientes para las ACP, encontrando diferencias significativas por grupos de edad $(\mathrm{p}=0,001)$. Del total de varones, un $23,6 \%$ eran dependientes para las ACP, presentando diferencias significativas por grupos de edad $(\mathrm{p}=0,002)$.

El apoyo informal disponible mostraba, que un $78,6 \%$ de las personas entrevistadas tenían una persona de apoyo principal que era un familiar de primer grado, un 7,4\% contaba con un familiar de segundo grado, un $4,6 \%$ disponía de otras personas no familiares y un 9,4 manifestaba que no disponía de apoyo. Un 24,9\% tenía apoyo compartido. Un 33,9\% recibían ayuda para las ACP. El $27,6 \%$ de la ayuda provenía de un familiar que convive, $1,1 \%$ de voluntariado y 1,4 del servicio de ayuda a domicilio.

Respecto a la ayuda que les podrían prestar en el futuro, 65,6\% contaba con el apoyo de una persona todo el tiempo necesario, al $10,4 \%$ le podían prestar ayuda durante un corto periodo de tiempo, al $11,5 \%$ ocasionalmente, y 9,0\% no contaba con ayuda de nadie. Cuando se preguntaba si tenían vecinos a los que les pudieran pedir un favor, $16,2 \%$ de las personas decían no tener a nadie, $29,6 \%$ de las personas respondían que a una persona o familiar, $20,7 \%$ podrían dirigirse a dos o tres personas y $33,2 \%$ a cuatro personas como mínimo.

Se realizó un análisis bivariante para estudiar la existencia de diferencias en el nivel de dependencia para las ACP (Tabla 1) según la edad, el sexo, situación de convivencia, nivel de estudios, ocupación, salud percibida, depresión y la existencia de apoyo informal para las ACP. El porcentaje de personas dependientes para las ACP aumentó con la edad y fue mayor en las mujeres $(p<0,001)$. La relación entre nivel de instrucción y dependencia en ACP presenta un gradiente. Aquellos con menos de primaria presentan mayor dependencia que los que llegaron a la secundaria, pero la asociación no alcanza significación estadística probablemente debido al pequeño tamaño de nuestra muestra. Un $26,0 \%$ de las personas que vivían solas eran dependientes y un $38,8 \%$ de las personas que valoraban su salud como regular y un $82,9 \%$ de las que opinaban que mala y muy mala también lo eran, existiendo diferencias estadísticamente significativas $(\mathrm{p}<0,001)$. Un 53,2\% y un $70,6 \%$ de las personas que presentaban depresión leve o establecida, respectivamente, eran dependientes $(\mathrm{p}<0,001)$. Respecto al apoyo que reciben las personas en función de su dependencia, el 12,5\% de las personas que no reciben apoyo son dependientes para 
Tabla 1

Relación de la capacidad funcional para las ACP con variables socioeconómicas y de apoyo informal en personas mayores de 75 años. Análisis bivariante

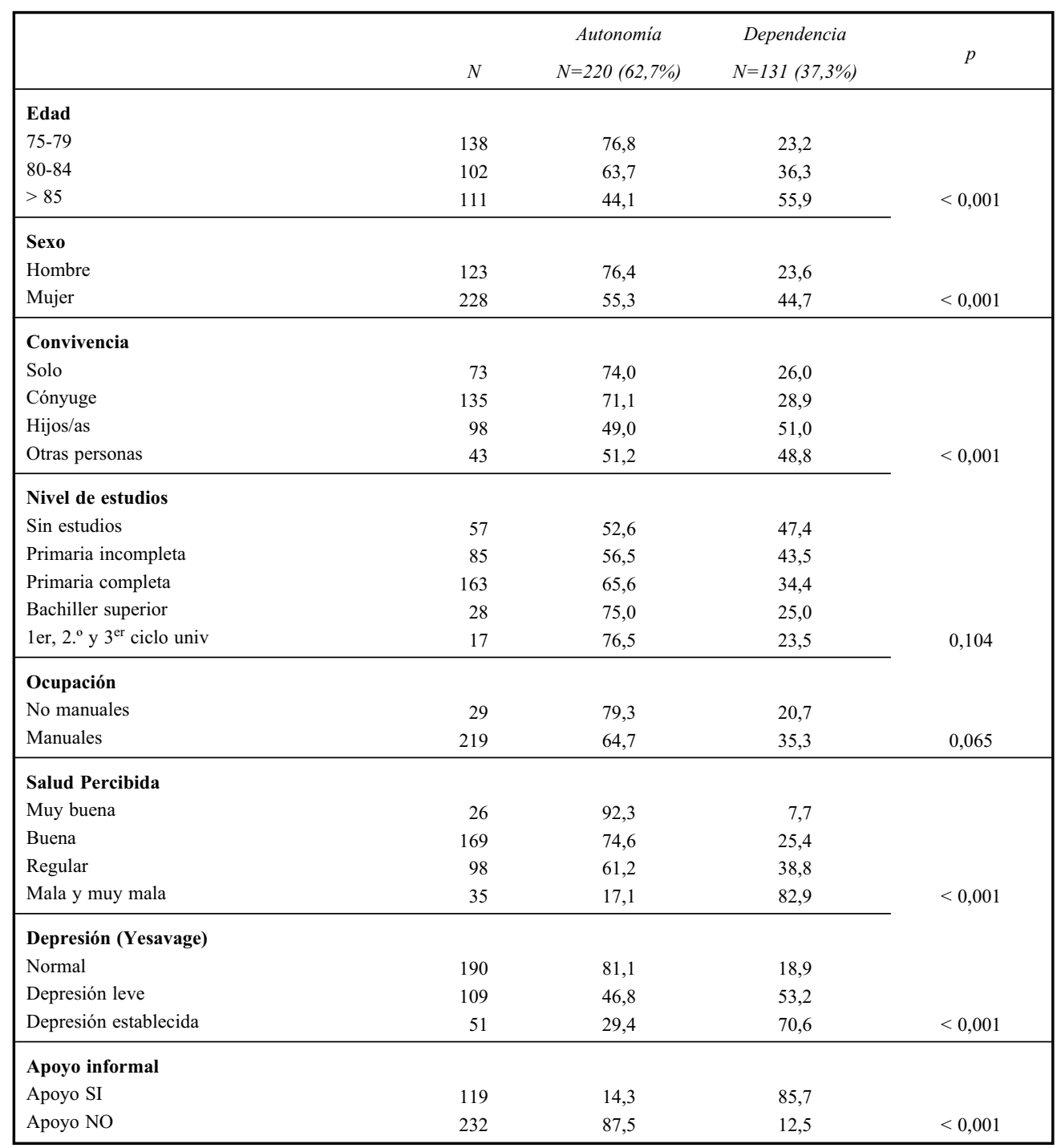

las ACP $(\mathrm{p}<0,001)$, y un $14,3 \%$ de personas que no lo necesitan lo reciben.

Se realizó un análisis de regresión logística tomando como variable dependiente la dependencia para las ACP. Las variables independientes se incluyeron en dos etapas, en primer lugar las variables antecedentes, edad, sexo y educación y en segundo lugar, el estado de salud y la convivencia. Las personas mayores de 85 años, las mujeres y las personas con menor nivel educativo tienen mayor probabilidad de ser dependientes respecto de los menores de 85 años, los hombres y los 
que tienen estudios superiores. Al introducir las variables de convivencia y salud en el modelo, la variable que mejor predice la situación de dependencia es la percepción de salud, una mala percepción de salud implica tener una mayor probabilidad de ser dependiente para las ACP. Vivir solo está asociado a la autonomía y aquellos con síntomas de depresión leve o establecida tienen mayor probabilidad de ser dependientes. La asocia-
$55,6 \%$ de las mujeres que viven solas no tienen cubiertas sus necesidades para las ACP $(p<0,001)$, siendo similar el porcentaje en aquellos que viven acompañados. Un $25,0 \%$ de los que tienen el nivel de primaria completa no tienen cubiertas sus necesidades y el $28,9 \%$ de los que perciben su salud como buena o regular, los que tienen una mala percepción de salud tienen mejor cubiertas sus necesidades. El 41,7\% de los que no pre-

Tabla 2

Estimación de las razones de ventaja (OR) de las variables asociadas a la dependencia en actividades del cuidado personal. Resultados del ajuste de la regresión logística multivariante

\begin{tabular}{|c|c|c|c|c|c|c|}
\hline & \multicolumn{3}{|c|}{ Modelo I } & \multicolumn{3}{|c|}{ Modelo II } \\
\hline & $O R$ & $p$ & IC $95 \%$ & $O R$ & $p$ & IC $95 \%$ \\
\hline \multicolumn{7}{|l|}{ Edad } \\
\hline $80-84 / 75-79$ & 1,81 & 0,050 & $1,001-3,307$ & 1,55 & 0,219 & $0,771-3,116$ \\
\hline$>85 / 75-79$ & 3,33 & $<0,001$ & $1,851-5,977$ & 3,79 & $<0,001$ & $1,869-7,668$ \\
\hline \multicolumn{7}{|l|}{ Sexo } \\
\hline Mujer/varón & 2,58 & 0,001 & $1,496-4,457$ & 2,02 & 0,038 & $1,042-3,938$ \\
\hline \multicolumn{7}{|l|}{ Educación } \\
\hline$<$ Primaria/Bachiller & 2,05 & 0,090 & $0,895-4,693$ & 1,40 & 0,498 & $0,530-3,693$ \\
\hline Primaria/Bachiller & 1,29 & 0,542 & $0,567-2,944$ & 0,87 & 0,779 & $0,330-2,294$ \\
\hline \multicolumn{7}{|l|}{ Convivencia } \\
\hline Otros/Hijos & & & & 1,10 & 0,835 & $0,446-2,720$ \\
\hline Solo / Hijos & & & & 0,38 & 0,021 & $0,168-0,868$ \\
\hline Cónyuge /Hijos & & & & 0,69 & 0,334 & $0,326-1,463$ \\
\hline \multicolumn{7}{|l|}{ Salud percibida } \\
\hline Regular/buena & & & & 1,99 & 0,025 & $1,091-3,649$ \\
\hline Mala/buena & & & & 9,40 & $<0,001$ & $3,335-26,498$ \\
\hline \multicolumn{7}{|l|}{ Depresión } \\
\hline Dp. Leve/normal & & & & 2,78 & 0,001 & $1,503-5,141$ \\
\hline Establecida/normal & & & & 5,14 & 0,001 & $1,943-13,606$ \\
\hline
\end{tabular}

ción entre nivel de instrucción y dependencia se debilita al incluir las variables de estado de salud indicando que una baja posición socioeconómica lleva a una peor salud que a su vez conduce a la dependencia.

Asimismo se realizó un análisis bivariante tomando como variable dependiente las necesidades de cuidados no cubiertas para las ACP (Tabla 3). La prevalencia de necesidades no cubiertas entre las personas dependientes para las ACP es de un 22,1\%. Un $27,5 \%$ de las personas entre 75-84 años y un sentan depresión y el 6,5 de los que tienen una depresión establecida no tienen sus necesidades de apoyo para las ACP cubiertas.

La regresión logística tomando como variable dependiente las personas con necesidades no cubiertas se muestra en la Tabla 4. En el primer modelo las mujeres que viven solas tienen mayor riesgo de no tener cubiertas sus necesidades respecto de los que viven acompañados $(\mathrm{p}=0,017)$ y los que tienen nivel de estudios inferior presentan mayor probabilidad de necesidades no 
Tabla 3

Distribución de variables asociadas a las necesidades de apoyo no cubiertas para las ACP. Análisis bivariado

\begin{tabular}{|c|c|c|c|c|}
\hline & \multicolumn{4}{|c|}{ Necesidades de cuidados } \\
\hline & $N$ & $\begin{array}{c}\text { Cubiertas } \\
N=102(77,9 \%)\end{array}$ & $\begin{array}{c}\text { No cubiertas } \\
N=29(22,1 \%)\end{array}$ & $p$ \\
\hline \multicolumn{5}{|l|}{ Edad } \\
\hline $75-84$ & 69 & 72,5 & 27,5 & \\
\hline$>85$ & 62 & 83,9 & 16,1 & 0,086 \\
\hline \multicolumn{5}{|l|}{ Sexo } \\
\hline Hombre & 28 & 82,1 & 17,9 & \\
\hline Mujer acom. & 84 & 83,3 & 16,7 & \\
\hline Mujer sola & 18 & 44,4 & 55,6 & $<0,001$ \\
\hline \multicolumn{5}{|l|}{ Nivel de estudios } \\
\hline Primaria incompleta & 64 & 78,1 & 21,9 & \\
\hline Primaria completa & 56 & 75,0 & 25,0 & \\
\hline Bachiller y más & 11 & 90,9 & 9,1 & 0,508 \\
\hline \multicolumn{5}{|l|}{ Salud percibida } \\
\hline Buena & 45 & 71,1 & 28,9 & \\
\hline Regular & 38 & 71,1 & 28,9 & \\
\hline Mala y muy mala & 29 & 86,2 & 13,8 & 0,270 \\
\hline \multicolumn{5}{|l|}{ Depresión (Yesavage) } \\
\hline Normal & 36 & 58,3 & 41,7 & \\
\hline Depresión leve & 58 & 81,0 & 19,0 & \\
\hline Depresión establecida & 36 & 94,4 & 6,5 & 0,001 \\
\hline
\end{tabular}

Tabla 4

Estimación de las razones de ventaja (OR) de las variables asociadas a las personas con necesidades no cubiertas en ACP. Resultados del ajuste de la regresión logística multivariante

\begin{tabular}{|c|c|c|c|c|c|c|}
\hline & \multicolumn{3}{|c|}{ Modelo I } & \multicolumn{3}{|c|}{ Modelo II } \\
\hline & $O R$ & $p$ & IC $95 \%$ & $O R$ & $p$ & IC $95 \%$ \\
\hline \multicolumn{7}{|l|}{ Edad } \\
\hline$>85 /<84$ & 0,52 & 0,154 & $0,216-1,275$ & 0,57 & 0,277 & $0,208-1,566$ \\
\hline \multicolumn{7}{|l|}{ Sexo-convivencia } \\
\hline Sola /acompañada-o & 2,60 & 0,017 & $1,188-6,676$ & 2,46 & 0,037 & $1,058-5,718$ \\
\hline \multicolumn{7}{|l|}{ Educación } \\
\hline$<$ Primaria/Bachiller & 3,53 & 0,265 & $0,384-32,502$ & 5,15 & 0,172 & $0,491-54,061$ \\
\hline Primaria/Bachiller & 3,50 & 0,267 & $0,383-32,082$ & 6,40 & 0,125 & $0,596-68,799$ \\
\hline \multicolumn{7}{|l|}{ Salud percibida } \\
\hline Regular/buena & & & & 1,15 & 0,798 & $0,394-3,360$ \\
\hline Mala/buena & & & & 0,63 & 0,548 & $0,140-2,840$ \\
\hline \multicolumn{7}{|l|}{ Depresión } \\
\hline Dp. Leve/normal & & & & 0,28 & 0,027 & $0,094-0,868$ \\
\hline Establecida/normal & & & & 0,13 & 0,025 & $0,021-0,777$ \\
\hline
\end{tabular}


cubiertas que los de estudios superiores $(\mathrm{OR}=3,50)$. Al introducir las variables de salud en el modelo se mantiene la asociación de ser mujer y vivir sola con la presencia de necesidades no cubiertas y aumenta la probabilidad de no tener las necesidades cubiertas en el grupo de nivel de estudios inferior respecto a los de estudios superiores $(\mathrm{OR}=5,15$ y 6,40$)$. Los que tienen una mala percepción de salud tienen menos riesgo de tener las necesidades no cubiertas, y tener depresión aparece como un factor de protección para tener las necesidades cubiertas; los que no tienen síntomas depresivos tienen mayor riesgo de tener las necesidades de cuidados no cubiertas.

\section{DISCUSIÓN}

La dependencia en las ACP en mayores de 75 años afecta a más de un tercio de la población. Las mujeres, las personas muy mayores y aquellos que tienen una peor percepción de salud tienen mayor probabilidad de dependencia. Dos de cada diez de estas personas dependientes no tienen sus necesidades cubiertas. Estas personas son mujeres que viven solas, con depresión y con pocos recursos sociales y económicos.

Respecto al perfil sociodemográfico, el porcentaje de personas que viven solas coincide con el estudio de Barcelona ${ }^{12}$ aunque es inferior al proyecto $\mathrm{ANCO}^{27}$, quizá por el diferente intervalo de edad estudiado. Las profesiones más frecuentes se han concentrado en el grupo de «sus labores», trabajos semi-cualificados y no cualificados, constituyendo estos grupos hasta el $77,2 \%$ del total. En nuestra población, un 40,6\% no llegan al nivel de estudios primarios completos, siendo este porcentaje relativamente inferior al presentado en otros estudios, de un $53 \%$ y un $78 \%$ respectivamente ${ }^{27,28}$. El perfil de las personas de nuestro estudio se asocia con una persona, casada o viuda, que vive principalmente en su domicilio, con el cónyuge o los hijos, y que tiene estudios primarios completos o incompletos. La clase social, medida a través de la ocupación, confirma que es una zona con un $2,9 \%$ de personas con profesiones asociadas a estudios universitarios y un $48,7 \%$ como trabajadores manuales semi-cualificados y no cualificados. La categoría amas de casa no figura en la clasificación y ha sido incluida como otra categoría.

Un $10,6 \%$ de las personas entrevistadas opina que su salud es mala o muy mala, porcentaje algo mayor que el presentado por Escudero et al. ${ }^{29}$ de un $4 \%$, o por Alonso en personas mayores de 65 años que lo observa en un $4,2 \%$ en varones y $7,5 \%$ en mujeres ${ }^{30}$.

El porcentaje de personas dependientes para las ACP varía según los estudios desde un $15,5 \%$ en el estudio de Leganés ${ }^{31}$, en mayores de 65 años, a un $27 \%{ }^{29}$ o un $29,2 \%$ a $34,8 \%{ }^{2}$ en mayores de 74 y 75 años respectivamente. En el nuestro es de un 37,3\%, bastante superior al de Leganés, probablemente por referirse a la población mayor de 75 años. La dependencia aumenta con la edad, independientemente del sexo, datos que coinciden con el estudio de Canarias ${ }^{32}$. El mayor porcentaje de dependencia para las ACP lo presentan los mayores de 85 años, tanto varones como mujeres.

Existen diferencias de género en la capacidad funcional, que indican que aunque las mujeres sobrevivan a los hombres, ellas pasan más tiempo en un estado de incapaci$\mathrm{dad}^{33}$. En el grupo de mujeres hay un porcentaje de dependencia para las ACP del doble respecto al grupo de los varones, esto coincide con otros estudios que señalan que las mujeres viven más pero tienen una peor calidad de vida que los varones. En el de Melzer et $\mathrm{al}^{34}$, en personas mayores de 65 años, el porcentaje de personas dependientes es $11 \%$ en varones y $19 \%$ en las mujeres. En el de Alonso et al ${ }^{29}$ los porcentajes se aproximan más al de nuestro estudio con un $31,8 \%$ en varones y $44,5 \%$ en mujeres. $\mathrm{La}$ asociación encontrada entre la dependencia para las ACP con la edad, ser mujer y convi- 
vencia, coincide con los presentados en el proyecto $\mathrm{ANCO}^{27}$.

El apoyo informal recibido para el cuidado personal coincide con otros estudios ${ }^{28}$ y se realiza principalmente por los familiares y prácticamente nada por el voluntariado o servicios formales. En este mismo estudio un $5 \%$ no disponen de cuidador, en el de Vigo un 6,7\% tiene una red social deteriorada $\mathrm{o}$ inexistente ${ }^{26}$ mientras que en nuestro estudio la no disponibilidad de apoyo asciende a un $9,4 \%$ de las personas entrevistadas.

Otros trabajos ${ }^{12}$ han estimado que un $43 \%$ de personas mayores de 60 años disponían de apoyo social. En mayores de 75 años, un $8,8 \%$ de los varones y el $8,9 \%$ de las mujeres tenían necesidad de apoyo para el cuidado personal. En el estudio de Leganés, en mayores de 65 años, el 18\% de los mayores reciben ayuda para las $\mathrm{ACP}^{35}$. El apoyo recibido en nuestro estudio o la necesidad de apoyo aumenta con la edad de las personas, datos que coinciden con los presentados por Sheterly $^{36}$.

Un $22,1 \%$ de las personas dependientes se encuentran con las necesidades de apoyo no cubiertas, siendo tres veces mayor el porcentaje en las mujeres que viven solas respecto a los que viven acompañados, tanto hombres como mujeres. Hay estudios que estiman que un $20,7 \%$ de los que necesitan algún tipo de ayuda para una ACP no la tienen cubierta, encontrando relación con vivir solo, mala situación socioeconómica y elevado número de ACP para las que se es dependiente $^{16,17,20}$, datos que coinciden con los encontrados en nuestro estudio.

Los que tienen un nivel de estudios inferior tienen un mayor riesgo de no tener cubiertas sus necesidades. Los que tienen una mala percepción de salud tienen mejor cubiertas sus necesidades aunque la percepción de salud no es un factor predictivo de necesidades no cubiertas. La depresión aparece como un factor de protección frente a las necesidades no cubiertas y los que no tienen síntomas depresivos tienen mayor riesgo de no tener cubiertas sus necesidades de cuidados.

Es importante destacar el elevado número de personas que no cuentan con apoyo; una de cada cinco personas mayor de 75 años con dependencia para las ACP no tienen cubiertas sus necesidades. Estas actividades hacen referencia a las actividades básicas y para medir su ayuda se ha tenido en cuenta que la ayuda se realiza diariamente o tres a cuatro veces por semana.

Las personas $>$ de 85 años tienen mejor cubiertas las necesidades de apoyo para las ACP mientras que ser mujer y estar sola es un marcador de riesgo de necesidades no cubiertas. Es también destacable que la salud percibida, un indicador de necesidad de servicios de salud, no está asociada a las necesidades de cuidados personales no cubiertas.

La comparación de resultados en los estudios sobre personas mayores plantea dificultades en su comparación por los diferentes grupos de edad que se consideran, y las distintas escalas de valoración de la autonomía utilizadas.

La elección de la tarjeta sanitaria limita la población a las personas con prestación de la seguridad social, y no permite eliminar las personas que están institucionalizadas. Esto unido a la alta frecuencia de traslados de domicilio, supone contactar un amplio número de personas, hecho muy frecuente en la población mayor, datos todos ellos que coinciden con los del estudio realizado en $\mathrm{Vigo}^{26}$. La muestra seleccionada ha sido del doble de mujeres que de hombres, ya que en la población de base se manifestaba esa misma característica y fue una de las variables de estratificación.

El carácter transversal del estudio no permite establecer asociaciones causales entre las variables independientes y sus efectos sobre la dependencia y las necesidades de 
cuidados no cubiertas. Por ejemplo, en este trabajo no se puede saber si las personas al encontrarse deprimidas tienen ayuda porque quizá la demandan más y la familia se moviliza para ayudarlas o si por el contrario, el hecho de recibir ayuda para los cuidados personales lleva a la depresión.

El modelo de atención a las personas mayores en España debería plantearse desarrollar medidas para evaluar las necesidades no cubiertas en población mayor dependiente, ya que nuestro modelo de cuidados tiene un sólido soporte en el apoyo informal. Los efectos negativos de la presencia de necesidades no cubiertas unido a los efectos positivos que tiene la presencia de apoyo, ya mencionados anteriormente, deberían ser suficientes para justificar el desarrollo de programas necesarios para cubrir estas necesidades. Es necesario realizar estudios que ayuden a definir el concepto de necesidades no cubiertas, individualizando para cada una de las ACP para la que no se dispone de apoyo, si se quiere conocer mejor la dinámica de los cuidados en el domicilio y los servicios que se necesitan. Sin ello no será posible estimar los servicios de apoyo que se deben integrar para que la población mayor dependiente reciba una atención y cuidados de calidad.

\section{AGRADECIMIENTOS}

Al CONSI+D (Gobierno de Aragón) por la financiación del proyecto, al equipo del CS Sta. Lucía por su colaboración y a las personas que respondieron a los cuestionarios

\section{BIBLIOGRAFÍA}

1. Kalache A. Active ageing makes the difference. Bull World Health Org 1999; 7: 299.

2. Winblad I, Jaaskelainen M, Kivela SL, Hiltunen P, Laippala P. Prevalence of disability in three birth cohorts at old age over time spans of 10 and 20 years. J Clin Epidemiol 2001; 54(10): 1019-24.

Rev Esp Salud Pública 2002, Vol. 76, N. ${ }^{\circ} 3$
3. Kivinen P, Sulkava R, Halonen P, Nissinen A. Self-reported and performance-based functional status and associated factors among elderly men: the Finnish cohorts of the seven countries study. J Clin Epidemiol 1998; 51: 1243-1252.

4. Hoeymans N, Feskens E, van den Bos AM, Kromhout D. Measuring functional status: cross-sectional and longitudinal associations between Performance and Self-Report (Zupten Elderly Study 1990-1993) J Clin Epidemiol 1996; 49: 1103-1110.

5. Santos-Eggimann B, Zobel F, Clerc Bérod A. Functional Status of Elderly Home Care Users: Do subjects, Informal and Professional Caregivers Agree? J Clin Epidemiol 1999; 52: 181-186.

6. Barón López de Roda A. Apoyo social: definición. Jano 1990; XXXVIII: 1086-1097.

7. Flórez Lozano JA, Valdés Sánchez C, Pérez Fernández $\mathrm{H}$, Hurtado Alvarez $\mathrm{P}$, Gómez Martín MP. Valor terapéutico del apoyo social en el anciano. Geriatrika 1992; 8: 17-27.

8. Branch LG, Jette AM. Elders' use of informal long-term care assistance. The Gerontologist 1983; 23(1): 51-56.

9. Organización Panamericana de la Salud. Salud de la población: conceptos y estrategias para políticas públicas saludables; «La perspectiva canadiense» Traducido por OPS, División Salud y Desarrollo Humano. Washington D.C.: OPS, 2000.

10. Pennix B, van Tiburg Th, Kriegsman D, Deeg D, Boeke A, van Eijik Th. Effects of social support and personal coping resources on mortality in older age: the longitudinal aging study Amsterdam. Am J Epidemiol 1997; 146: 510-519.

11. Hanson BS, Isacsson S-O, Janzon L, Lindell S-E. Social network and social support influence mortality in elderly men. Am J Epidemiol 1989; 130: 100-111.

12. Ferrando J, Nebot M, Borrell C, Egea L. Apoyo social y estado de salud percibido en población no institucionalizada de más de 60 años. Gac Sanit 1996; 10: 174-182.

13. Fratiglioni L, Wang H-X, Ericsson K, Maytan M, Winblad B. Influence of social network on ocurrence of dementia: a community-based longitudinal study. Lancet 2000; 355: 1315-19.

14. Barefoot JC, Brummett BH, Clapp-Channing EN, Siegler IC, Vitaliano PP, Williams RB, Mark DB. Moderators of the effect of social support on de- 
pressive symtoms in cardiac patients. Am J Cardiol 2000; 86: 438-442.

15. Seeman, TE, Albert M, Lusuignolo TN, \& Berkman L. Social relationships, social support, and patterns of cognitive aging in healthy, high-functioning older adults: MacArthur studies of successful aging. Health Psychology 2001; 20: 243-255.

16. Dessai MM, Lentzner HR, Weeks JD. Unmet need for personal assistance with activities of daily living among older adults. Gerontologist 2001; 41(1): 82-8.

17. Kennedy J. Unmet and undermet need for acitivities of daily living and instrumental acitivities of daily living assistance among adults with disabilities: estimates from the 1994 and 1995 disability follow-back surveys. Med Care 2001; 39(12): 1305-12.

18. Kent RM, Chandler BJ, Barnes MP. An epidemiological survey of the health needs of disabled people in a rural community. Clin Rehabil 2000; 14(5): 481-90.

19. Allen SM, Mor V. The prevalence and consequences of unmet need. Contrasts between older and younger adults with disability. Med Care 1997; 35(11): 1132-48.

20. Williams J, Lyons B, Rowland D. Unmet long-term care needs of elderly people in the community: a review of the literature. Home Health Care Serv Q 1997; 16(1-2): 93-119.

21. De Veer AJ, de Bakker DH. Measuring unmet needs to asses the quality of home health care. Int J Qual Health Care 1994; 6(3): 267-74.

22. Mor V, Allen SM, Siegel K, Houts P. Determinants of need and unmet need among cancer patients residing at home. Health Serv Res 1992; 27(3): 337-60.

23. Ministerio de Asuntos Sociales. Cuidados en la vejez. El apoyo informal. Madrid: Ministerio de Asuntos Sociales Instituto Nacional de Servicios Sociales (INSERSO), 1995.

24. Grupo de trabajo de la Sociedad Española de Epidemiología. La medición de la Clase Social en Ciencias de la Salud. Barcelona: SG Editores, 1995.

25. Katz S, Ford AB, Moskowitz RW, Jackson BA, Jaffe MW, Cleveland JMA. Studies of illness in the Aged. JAMA 1963; 185: 94-99.

26. Eiroa Patiño P, Vázquez-Vizoso FL, Veras castro R. Discapacidades y necesidades de servicios en las personas mayores detectadas en la encuesta de salud OARS-Vigo. Med Clin(Barc) 1996; 106: 641-648.

27. Espejo Espejo J, Martínez de la Iglesia J, Aranda Lara JM, Rubio Cuadrado V, Enciso Bergé I, Zunzunegui pastor MV, Pérula Torres L, Fonseca del Pozo FJ. Capacidad funcional en mayores de 60 años y factores sociosanitarios asociados (proyecto ANCO). Atención Primaria 1997; 20: 21-35.

28. García Bermejo S, Lebuke Angulo K, Andikoetxea Agorria A, Solar Barruetabeña M, Olaskoaga Arrate A. Características socieconómicas, problemas y necesidades de salud de los pacientes crónicos domiciliarios. Atención Primaria 1997; 20: 230-236.

29. Escudero Sánchez C, García Carmona R, Ibáñez Colás A, López López MA, Hidalgo García-Consuegra MA, Pérez Juárez A, Salán García M, Utrilla Bermejo F. Déficit cognitivo, prevalencia y factores asociados en la población mayor de 74 años. Atención Primaria 1999; 24: 326-331.

30. Alonso J, Orfila F, Ruigómez A, Ferrer M, Antó JM. Unmet health care needs and mortality among spanish elderly. Am J Publ Health 1997; 87: $365-370$.

31. Beland F, Zunzunegui MV. El perfil de las incapacidades funcionales en las personas mayores. Rev Gerontol 1995; 5: 232-244.

32. Ania Lafuente BJ, Suárez Almenara JL, Guerra Hernández L, Santana Santana AJ, Acosta Morales CD, Saavedra Rodríguez JM. Vejez saludable e incapacidad funcional en la población anciana de Canarias. Rev Esp Salud Pública 1997; 71: 161-171.

33. Dunlop DD, Hughes SL, Manheim LM. Disability in activities of daily living: patterns of change and a hierarchy of disability. Am J Publ Health 1997; 87: 378-383.

34. Melzer D, McWilliams B, Brayne C, Johnson T, Bond J. Profile of disability in elderly people: estimates from a longitudinal population study. BMJ 1999; 318: 1108-11.

35. Beland F, Zunzunegui MV. La ayuda recibida por las personas mayores. Rev Gerontol 1995; 5: 294-308.

36. Sheterly SM, Morgenstern EN, Grigsby J, Hamman RF. Higher instrumental activities of daily living disability in Hispanics Compared with non hispanic whites in rural Colorado. Am J Epidemiol 1998; 147: 1019-1027.

Rev Esp Salud Pública 2002, Vol. 76, N. ${ }^{\circ} 3$ 